\title{
Vanishing vessels aboding pulmonary disease: a role for VEGFR2
}

\author{
Liza Botros, Anton Vonk Noordegraaf and Jurjan Aman
}

\begin{abstract}
Affiliation: Dept of Pulmonary Diseases, Amsterdam University Medical Center, Location VU University Medical Center, Amsterdam, The Netherlands.

Correspondence: Jurjan Aman, Dept of Pulmonary Diseases, Amsterdam University Medical Center, Location VU University Medical Center, De Boelelaan 1117, 1081 HV Amsterdam, The Netherlands. E-mail: j.amana amsterdamumc.nl
\end{abstract}

@ERSpublications

VEGFR2 has a pivotal and specific role in vascular homeostasis and VEGFR2 mutations provide robust clinical evidence for direct involvement of a disturbed vasculature in a wide variety of lung diseases http://bit.ly/383ixTb

Cite this article as: Botros L, Vonk Noordegraaf A, Aman J. Vanishing vessels aboding pulmonary disease: a role for VEGFR2. Eur Respir J 2020; 55: 2000326 [https://doi.org/10.1183/13993003.00326-2020].

There is growing recognition for the involvement of blood vessels in pulmonary diseases beyond the classical scope of pulmonary vascular medicine. As an example, reports have described a vascular phenotype of COPD, or have proposed a vanishing pulmonary capillary syndrome to underly emphysema $[1,2]$. Although the number of clinical and epidemiological studies supporting such a vascular hypothesis of pulmonary disease is steadily growing, biological underpinning in clinical studies remains to be provided.

In this issue of the European Respiratory Journal, EYRIES et al. [3] make an unprecedented step in establishing the link between vascular stability and pulmonary disease. Analysing the vascular endothelial growth factor receptor 2 (VEGFR2/KDR) gene in patients with pulmonary arterial hypertension (PAH), they report on two index cases from two separate families, and demonstrate that heterozygous mutations in the VEGFR2/KDR gene cosegregate with clinical features of emphysema and low diffusing capacity of the lung for carbon monoxide $\left(D_{\mathrm{LCO}}\right)$ in virtually all carriers, while interstitial disease and severe pulmonary arterial hypertension were observed in some. As the observed mutations in these families were predicted to be deleterious for protein function of VEGFR2, this study provides a causative link between vascular stability and a wide variety of pulmonary diseases in humans.

VEGFR2 is a transmembrane receptor that translates extracellular signals into changes in cell behaviour (figure 1a). Its predominant ligand in blood vessels is vascular endothelial growth factor-A (VEGF-A), whereas lymphatic VEGFR2 may also bind VEGF-C, a lymph specific growth factor [4, 5]. Genetic knockout of VEGFR2 (KDR) in mouse models is embryonically lethal at day 8-9 due to impaired vasculogenesis and angiogenesis [6,7], indicative of the critical importance of VEGFR2 in vascular development. In the lung, VEGFR2 is robustly and almost exclusively expressed in the vascular endothelium, both during development [8] and in the adult lung [4]. Due to lethality early in embryonic development, it is unclear how genetic knockout of VEGFR2 affects lung development. Yet, it is generally acknowledged that VEGFR2 is critical for lung development and specifically the alveolo-capillary interface. VEGF-A excreted by budding alveoli binds VEGFR2 of angiogenic endothelium, so that sprouting vessels follow alveolar buds and match perfusion to ventilation in the developing lung $[9,10]$. Pharmacological inhibition of angiogenesis (VEGFR2 inhibitor) at this stage decreases alveolarisation [11]. Although there 
a)

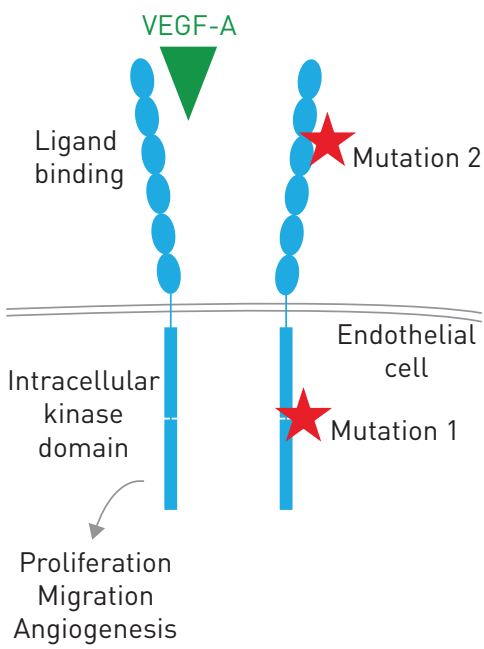

b)

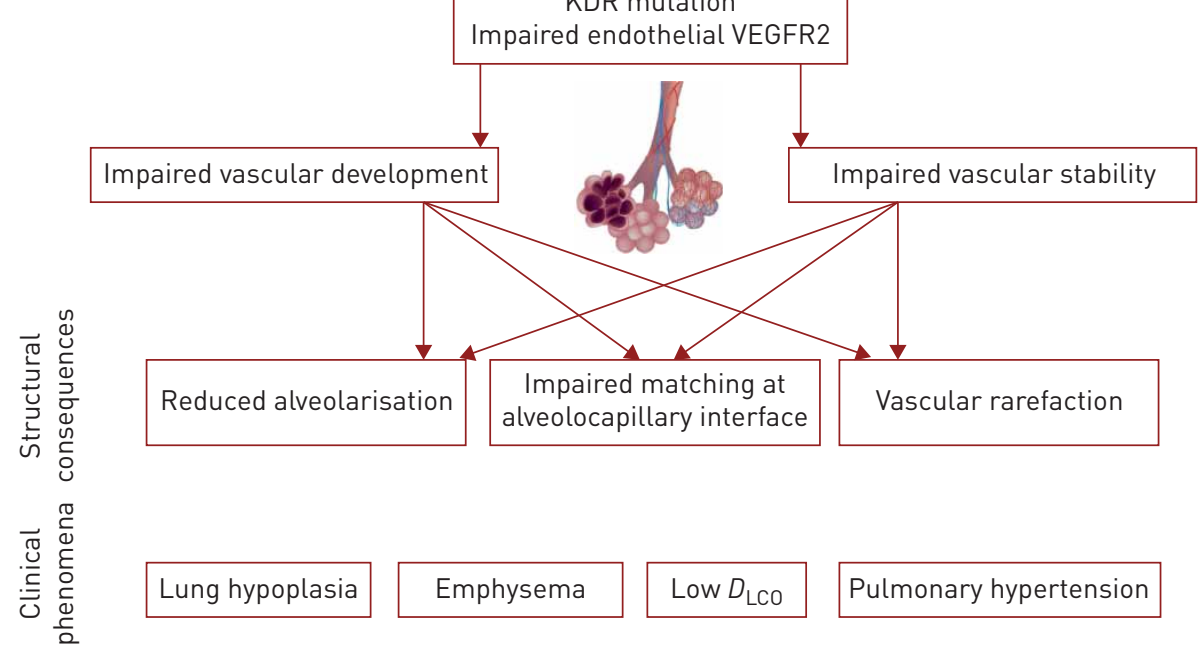

FIGURE 1 a) The ligand vascular endothelial growth factor (VEGF)-A binds to VEGF receptor 2 (VEGFR2) on endothelial cells, leading to homodimerisation and activation of the receptor. This leads to an intracellular signalling of proliferation, migration and angiogenesis. The mutations described by EYRIES et al. [3] are located at the intracellular tyrosine kinase domain in family 1, and at the extracellular ligand binding site in family 2. Both mutations are predicted to severely impair the function of the receptor. b) VEGFR2 function and signalling is of the critical importance for vascular development and maintenance. When VEGFR2 expression is reduced in the vascular endothelium of the lung, a reduced alveolarisation, impaired alveolocapillary interface and a loss of vessels occurs. This gives rise to a number of clinical phenotypes including emphysema, low diffusing capacity of the lung for carbon monoxide $\left(D_{\mathrm{LCO}}\right)$ and pulmonary hypertension.

is evidence for low expression of VEGFR2 in non-endothelial cells in the lung, such as type II alveolar epithelial cells and haematopoietic progenitor cells $[4,8,12]$, the function of VEGFR2 in these cell types is unclear due to lack of conditional knockout studies.

Animal studies in the adult lung, using either conditional genetic knockout or chronic pharmacological inhibition, demonstrated that vascular VEGFR2 is required for maintenance and repair of the lung. In contrast to constitutional knockout, conditional knockout of endothelial VEGFR2 at adult age is not lethal and does not carry a phenotype unless challenged [13, 14]. Regenerative lung alveolarisation, however, was severely impaired in these mice after pneumonectomy [13], indicating that endothelial VEGFR2 orchestrates lung tissue regeneration. Chronic administration of the VEGFR2 inhibitor SU5416 to rats resulted in alveolar septal cell apoptosis, decreased alveolarisation, enlargement of the air spaces and emphysema [12]. Importantly, the emphysema in this rat model was paralleled by pruning of the pulmonary vascular tree, linking emphysema to vascular instability [12]. These animal data illustrate the pivotal role of endothelial VEGFR2 for development, maintenance and recovery of lung parenchyma, although the clinical relevance remains to be settled.

Against this background of VEGFR2 biology, the work of Eyries et al. [3] forms a final piece of the puzzle linking naturally occurring variation in VEGFR2 expression to clinical pulmonary phenomena in two index families. Given the critical dependence of the pulmonary vasculature on VEGFR2 for maintenance and stability, this study provides clinical proof that undermining vascular stability per se (by haplo-insufficiency of VEGFR2) results in a broad spectrum of pulmonary diseases, including pulmonary hypertension, emphysema and interstitial disease (figure 1b). Besides that, the study of EyrIES et al. [3] carries important implications for respiratory research at several levels. First, it puts a weighty argument in the ongoing debate on the role of VEGF/VEGFR signalling in pulmonary hypertension, which has evolved around the paradoxical findings that VEGF-A and VEGF receptors were reported to be upregulated in plexiform lesions in PAH on the one side [15, 16], and that blockade of VEGF-A [17] or VEGFR2 [18, 19] results in PAH. EYRIES et al. [3] provide evidence for the latter by showing that VEGFR2 haplo-insuffiency contributes to $\mathrm{PAH}$, indicating that upregulation of VEGF-A and receptors are secondary events, either by negative feedback or from local receptor rearrangement. Second, these observations strengthen the rationale for the use of the Sugen/hypoxia rat model as an animal model for pulmonary arterial hypertension. Besides recapitulation of important morphological characteristics of precapillary PAH, including plexiform lesions, the current study also shows that the Sugen/hypoxia model recapitulates pathophysiological mechanisms of precapillary PAH, i.e. reduced VEGFR2 expression. Third, the study provides mechanistic support for a vascular hypothesis of COPD/emphysema [20]. Although observational 
studies report pulmonary vascular remodelling early in COPD/emphysema [1], the underlying mechanisms remain incompletely understood. Together with fundamental studies showing that cigarette smoke reduces endothelial VEGFR2 expression [21], the strong cosegregation of VEGFR2 mutations with a low $D_{\text {LCO }}$ observed by EYries et al. [3] (six out of seven carriers had a $D_{\text {LCO }}<65 \%$ ) closes the loop, establishing loss of vascular integrity as significant player in COPD/emphysema.

Having said that, too many uncertainties remain to call it a closed chapter. A number of unanswered questions need clarification in future studies. A first question is, how the mutations described in the two families affect VEGFR2 function. The mutations described include a truncation mutation in exon 24, affecting the intracellular kinase domain (family 1), and a mutation in the donor splicing site of intron 7 , resulting in skipping of exon 7 and a protein truncation (family 2). Both mutations are predicted to severely impair the function of the translated product, either by impaired ligand binding or abolished intracellular signalling (figure 1a). Yet, since VEGFR2 undergoes homodimerisation [4], it remains to be determined whether the truncated products affect dimerisation and thereby have deleterious effects beyond haplo-insufficiency. Second, given the hereditary nature of the mutations and given the pivotal role of VEGFR2 in embryonic development and adult maintenance of the pulmonary vasculature, it is an intriguing question whether the observed phenomena result from impaired ante- and postnatal development versus impaired homeostasis and repair (vulnerability). Although this question holds true for all described phenomena it might be of particular interest for pulmonary hypertension, being observed in only three out of seven mutation carriers. This relatively low penetrance may suggest the requirement of a second hit and therefore point towards increased vulnerability rather than a developmental problem. Finally, given the wide array of clinical phenomena observed in the index patients (pulmonary hypertension, low $D_{\mathrm{LCO}}$ and interstitial disease) it remains unclear whether this type of pulmonary hypertension should be classified as group I or group III pulmonary hypertension. In particular, since the cosegregation of KDR mutation with low $D_{\mathrm{LCO}}$ (six out of seven) seems more complete than with pulmonary hypertension (three of seven). Further characterisation of the interstitial disease, e.g. by histological examination, may provide answers here.

Overall, the work of EYRIES et al. [3] identifies VEGFR2/KDR as novel gene associated with hereditary pulmonary hypertension. More important, given the pivotal and specific role of VEGFR2 in vascular homeostasis, it provides robust clinical evidence for direct involvement of a disturbed vasculature in a wide variety of lung diseases.

Conflict of interest: None declared.

\section{References}

1 Washko GR, Nardelli P, Ash SY, et al. Arterial vascular pruning, right ventricular size, and clinical outcomes in chronic obstructive pulmonary disease. A longitudinal observational study. Am J Respir Crit Care Med 2019; 200: $454-461$.

2 Hoeper MM, Vonk-Noordegraaf A. Is there a vanishing pulmonary capillary syndrome? Lancet Respir Med 2017; 5: 676-678.

3 Eyries M, Montani D, Girerd B, et al. Familial pulmonary arterial hypertension by KDR heterozygous loss of function. Eur Respir J 2020; 55: 1902165.

4 Voelkel NF, Vandivier RW, Tuder RM. Vascular endothelial growth factor in the lung. Am J Physiol Lung Cell Mol Physiol 2006; 290: L209-L221.

5 Tammela T, Alitalo K. Lymphangiogenesis: Molecular mechanisms and future promise. Cell 2010; 140: 460-476.

6 Yin LY, Wu YX, Ballinger CA, et al. Genomic structure of the human KDR/flk-1 7 gene. Mamm Genome 1998; 9: 408-410.

7 Shalaby F, Rossant J, Yamaguchi TP, et al. Failure of blood-island formation and vasculogenesis in Flk-1-deficient mice. Nature 1995; 376: 62-66.

8 Kaipainen A, Korhonen J, Pajusola K, et al. The related Flt4, Flt1, and Kdr receptor tyrosine kinases show distinct expression patterns in human fetal endothelial-cells. J Exp Med 1993; 178: 2077-2088.

9 Healy AM, Morgenthau L, Zhu X, et al. VEGF is deposited in the subepithelial matrix at the leading edge of branching airways and stimulates neovascularization in the murine embryonic lung. Dev Dyn 2000; 219: 341-352.

10 Aman J, Bogaard HJ, Vonk Noordegraaf A. Why vessels do matter in pulmonary disease. Thorax 2016; 71: 767-769.

11 Jakkula M, Le Cras TD, Gebb S, et al. Inhibition of angiogenesis decreases alveolarization in the developing rat lung. Am J Physiol Lung Cell Mol Physiol 2000; 279: L600-L607.

12 Kasahara Y, Tuder RM, Taraseviciene-Stewart L, et al. Inhibition of VEGF receptors causes lung cell apoptosis and emphysema. J Clin Invest 2000; 106: 1311-1319.

13 Ding BS, Nolan DJ, Guo P, et al. Endothelial-derived angiocrine signals induce and sustain regenerative lung alveolarization. Cell 2011; 147: 539-553.

14 Jang JY, Choi SY, Park I, et al. VEGFR2 but not VEGFR3 governs integrity and remodeling of thyroid angiofollicular unit in normal state and during goitrogenesis. EMBO Mol Med 2017; 9: 750-769.

15 Tuder RM, Chacon M, Alger L, et al. Expression of angiogenesis-related molecules in plexiform lesions in severe pulmonary hypertension: evidence for a process of disordered angiogenesis. J Pathol 2001; 195: 367-374.

16 Hirose S, Hosoda Y, Furuya S, et al. Expression of vascular endothelial growth factor and its receptors correlates closely with formation of the plexiform lesion in human pulmonary hypertension. Pathol Int 2000; 50: 472-479. 
17 McGee M, Whitehead N, Martin J, et al. Drug-associated pulmonary arterial hypertension. Clin Toxicol 2018; 56: 801-809.

18 Taraseviciene-Stewart L, Kasahara Y, Alger L, et al. Inhibition of the VEGF receptor 2 combined with chronic hypoxia causes cell death-dependent pulmonary endothelial cell proliferation and severe pulmonary hypertension. FASEB J 2001; 15: 427-438.

19 Le Cras TD, Markham NE, Tuder RM, et al. Treatment of newborn rats with a VEGF receptor inhibitor causes pulmonary hypertension and abnormal lung structure. Am J Physiol Lung Cell Mol Physiol 2002; 283: L555-L562.

20 Voelkel NF, Gomez-Arroyo J, Mizuno S. COPD/emphysema: the vascular story. Pulm Circ 2011; 1: 320-326.

21 Marwick JA, Stevenson CS, Giddings J, et al. Cigarette smoke disrupts VEGF(165)-VEGFR-2 receptor signaling complex in rat lungs and patients with COPD: morphological impact of VEGFR-2 inhibition. Am J Physiol Lung Cell Mol Physiol 2006; 290: L897-L908. 